\title{
Overuse - unnötige Behandlungen als Qualitätsproblem
}

\author{
Michelle Gerber ${ }^{a}$, Esther Kraft ${ }^{b}$, Christoph Bosshard ${ }^{c}$ \\ ${ }^{a}$ lic. phil. hum., wissenschaftliche Mitarbeiterin DDQ/SAQM; ' lic. rer. oec., Leiterin Abteilung DDQ; ${ }^{c}$ Dr. med., Vizepräsident der FMH, Departements- \\ verantwortlicher DDQ/SAQM
}

Die Abteilung Daten, Demographie und Qualität (DDQ) erstellt basierend auf wissenschaftlicher Literatur Grundlagenpapiere zu verschiedenen Themen im Bereich Qualität, die in der Schweizerischen Ärztezeitung veröffentlicht werden. Die FMH nimmt auf der Basis der erarbeiteten Grundlagen Stellung zum Thema und bringt diese Stellungnahme über das «Positionspapier der FMH» an die Öffentlichkeit. Nachfolgend werden das Grundlagenpapier sowie die Position der FMH zum Thema Overuse präsentiert.

Die Literatur findet sich
unter www.saez.ch
$\rightarrow$ Aktuelle Ausgabe oder
$\rightarrow$ Archiv $\rightarrow 2016 \rightarrow 7$.
*Aus Gründen der besseren
Lesbarkeit wird auf die
gleichzeitige Verwendung
männlicher und weib-
licher Sprachformen
verzichtet. Sämtliche
Personenbezeichnungen
gelten gleichwohl für
beiderlei Geschlecht.
Weitere Vorstösse erfolg-
ten unter anderem zu
hoher Kaiserschnittrate
[11], Mengenausweitungen
[12], unnötigen Operatio-
nen [13] oder unnötigen
Herzuntersuchungen [14].

«Ich fürchte mich davor, einer Frau eine Mammographie nicht zu empfehlen, die vielleicht später mit Brustkrebs wiederkommt und mich fragt, warum ich keines habe machen lassen. Deshalb empfehle ich das Screening, obwohl ich persönlich der Überzeugung bin, dass es nicht empfohlen werden sollte.» (Aussage einer Gynäkologin [1]*)

Die grundsätzliche Idee von Overuse ist in der Ärzteschaft seit je bekannt [2]: Gesundheitsfachpersonen sollen keine unnötigen oder sogar schädlichen medizinischen Leistungen erbringen, welche die Prognose für Patienten** nicht verbessern oder sogar verschlechtern. Viele Ärzte erachten Overuse als problematisch [3, 4]. So vertraten 51 Prozent der Schweizer Grundversorger 2015 die Meinung, dass Patienten zu viele medizinische Leistungen erhalten [5]. Overuse vermindert

\section{Zusammenfassung}

Der Begriff Overuse meint medizinische Leistungen, die für den Patienten keinen Nutzen haben oder deren Risiken den potentiellen Nutzen übersteigen. Unnötige Untersuchungen und Behandlungen können auch durch Überdiagnosen entstehen. Damit sind Diagnosen gemeint, welche gemäss Untersuchungen in Langzeitstudien bei Patienten nie zu Symptomen oder deren Tod geführt hätten. Der vorliegende Artikel gibt interessierten Fachpersonen einen Überblick über die wissenschaftliche Literatur zu Overuse, deren verschiedenen Einflussfaktoren und Lösungsansätze. Overuse vermindert die Qualität der medizinischen Versorgung und gefährdet die Patientensicherheit durch die Risiken der unnötigen Tests, Untersuchungen und Behandlungen. Neben mehr Forschung und einer Sensibilisierung der Ärzteschaft und Patienten für dieses Thema sollen fachspezifische, evidenzbasierte Empfehlungen zu unnötigen Leistungen entwickelt werden. die Qualität der medizinischen Versorgung. Wie alle Behandlungen können auch unnötige Behandlungen zu Nebenwirkungen und Komplikationen führen, bei risikoreichen Behandlungen manchmal sogar zum Tod. Unnötige Diagnosen lösen bei den Betroffenen ausserdem viele Sorgen, Ängste und Stress aus [6], die teilweise über längere Zeit anhalten können [7, 8]. Ausserdem werden dadurch weitere diagnostische Untersuchungen und Behandlungen nötig, welche die Zeit von Patienten und Ärzten in Anspruch nehmen und von einer emotionalen Anspannung geprägt sind. Aufgrund solcher unnötiger Diagnosen kann es sein, dass Patienten keine neue Zusatzversicherung mehr abschliessen können [6].

In der Politik und Öffentlichkeit wurde das Thema Overuse besonders in den letzten Jahren aktuell und publik, vor allem durch Publikationen wie das Positionspapier zu nachhaltiger Medizin der Schweizerischen Akademie der Medizinischen Wissenschaften SAMW [9], die Smarter Medicine-Initiative (siehe Abschnitt Lösungsansätze) sowie verschiedene politische Vorstösse (z.B. Motion «Less is more - Wie steht es in der Schweiz mit dem Überkonsum von medizinischen Leistungen?» [10]) $)^{1}$. Der Bundesrat forderte im Bericht Gesundheit2020 [15] eine «Reduktion von nicht wirksamen und nicht effizienten Leistungen sowie Medikamenten und Verfahren, um die Qualität zu erhöhen und die Kosten zu reduzieren".

Der vorliegende Artikel thematisiert Overuse als Qualitätsproblem. Ziel des Beitrags ist es, eine Übersicht zum Thema Overuse zu geben und insbesondere die Entstehungsbedingungen sowie mögliche Lösungs- 
ansätze aufzuzeigen. Er soll die nötigen wissenschaftlichen Grundlagen liefern für Ärzteorganisationen, Ärzte und weitere Interessierte, die sich vertieft mit Overuse auseinandersetzen möchten.

Neben Overuse ist auch Underuse ${ }^{2}$ eine Herausforderung für das Schweizer Gesundheitssystem. Obwohl diese beiden Themen in Bezug zueinander stehen [1820], sind die Ursachen für die Entstehung und entsprechend die Lösungsansätze nicht immer die gleichen. Folglich befasst sich der vorliegende Artikel nur mit Overuse. Aufgrund der Ausrichtung des Artikels auf Qualitätsaspekte werden Auswirkungen auf die Gesundheitskosten nicht diskutiert, und auf verwandte Themen wie Polypharmazie [21] und Behandlung am Lebensende $[9,22]$ wird nicht eingegangen.

\section{Was ist Overuse?}

Der Begriff Overuse (dt. Überversorgung) wird im vorliegenden Grundlagenpapier folgendermassen verstanden: Versorgung mit einer umschriebenen medi-

\section{Subklinische Krankheiten sind bei vielen Menschen vorhanden, ohne dass sie je Symptome entwickelt hätten.}

zinischen Diagnostik oder Behandlung, die für den Patienten keinen Nutzen hat oder deren Risiken den potentiellen Nutzen übersteigen [16, 23-25]. In der Literatur werden auch verwandte Begriffe wie z.B. Überbehandlung [26], Überdiagnosen [26] oder quartäre Prävention [27] verwendet. Overuse weist einen engen Bezug zur Indikationsqualität auf, welche «die Angemessenheit und Notwendigkeit medizinischer Interventionen» [28] umfasst. Zudem verletzt Overuse einerseits das Prinzip der Effektivität [29], wonach Leistungen basierend auf wissenschaftlichen Erkenntnissen nur bei Patienten erbracht werden, die davon profitieren können, und andererseits das Prinzip der Zweckmässigkeit [30], nach welchem der erwartete Gesundheitsnutzen die erwarteten negativen Konsequenzen einer Leistung übersteigt.

Underuse meint eine einer umschriebenen medizinischen Diagnostik oder Behandlung, welche einen hinreichend gesicherten Nettonutzen hat $[16,17]$.

3 Für eine Diskussion der Qualität von Guidelines siehe Hostettler et al. [31] 4 In der Praxis kann allerdings nicht immer klar zwischen Überdiagnose und falsch positiver Diagnose unterschieden werden [6]. propriateness Method (RAM), welche die beste vorhandene wissenschaftliche Evidenz mit Expertenmeinungen kombiniert. Es können auch Choosing Wisely-Listen (vgl. Abschnitt Lösungsansätze) oder Guidelines ${ }^{3}$ verwendet werden. Letztere müssen aber für verschiedene klinische Szenarien festgelegt sein. Somit wird deutlich: Mit der Weiterentwicklung von wissenschaftlichen Erkenntnissen und medizinischen Techniken ändern auch die medizinischen Leistungen, die als Overuse definiert werden.

Für die Messung der Häufigkeit von unnötigen Leistungen müssen genügend klinische Details erhoben werden, um die Angemessenheit der Behandlung oder Untersuchung beurteilen zu können [16, 24, 32]. Studien zur Untersuchung von Overuse sind sehr aufwendig. Das Institute of medicine [29], Korenstein et al. [24] und Kale et al. [16] dokumentieren verschiedene, wenn auch eine eingeschränkte Anzahl von sehr spezifischen Leistungen in Übersichtsartikeln in den USA. Am meisten Studien existieren in den Bereichen Antibiotikagebrauch und kardiologische Prozeduren. Eine Schweizer Studie belegt, dass bei 34 Prozent der Patienten unangemessene diagnostische Koronarangiographien zur Abklärung von kardiovaskulären Erkrankungen durchgeführt wurden. Leitlinien sehen diese invasive Untersuchung (ein Katheter wird bis ins Herz geschoben) nur vor, wenn vorgängig ein auffälliges Resultat bei einer nicht-invasiven Untersuchung vorliegt. Denn in beinahe 3 Prozent der Fällen treten schwerwiegende Komplikationen auf, wie z.B. ein Schlaganfall $[33,34]$.

Viele Patienten (und zum Teil auch Ärzte) glauben intuitiv, dass mehr und neuere medizinische Leistungen ein Qualitätsanzeichen sind.

Overuse entsteht auch durch die Behandlung von $\mathrm{Pa}$ tienten, bei denen unnötige Diagnosen gestellt wurden. Überdiagnosen werden definiert als Diagnosen, die einer Person keinen Nutzen bringen, weil die diagnostizierte Erkrankung während der verbliebenen Lebenszeit weder zum Tod führte noch Symptome auftraten [35-37]. Überdiagnosen werden auch als Pseudokrankheit, klinisch irrelevante Diagnose oder gutartige Abnormalität bezeichnet [35]. Überdiagnosen sind abzugrenzen von Diagnosefehlern, d.h. falsch gestellten Diagnosen oder falsch positiven Diagnosen ${ }^{4}$, bei denen ein Testresultat fälschlicherweise auf das Vorliegen einer Erkrankung hindeutet [36, 37]. Hingegen ist bei einer Überdiagnose die Diagnose zwar korrekt gestellt, aber sie hat keinen Nutzen für den Patienten. Überdiagnosen entstehen z.B. durch Screenings (siehe Abschnitt Wie kommt es zu Overuse?). Denn 
nicht alle entdeckten Tumore entwickeln sich zu einem tödlichen Krebs. Tumore können sich auch zurückbilden, unverändert bleiben oder nur ganz langsam wachsen. Manchmal entwickelt sich innerhalb kürzester Zeit ein tödlicher Krebs, so dass dieser beim Screening gar nicht entdeckt wird, weil er danach entstanden ist [6, 37]. Problematisch bei Überdiagnosen ist, dass die Patienten in der Regel (unnötigerweise) weiter untersucht und behandelt werden und so teilweise beträchtlichen Risiken ausgesetzt sind [36]. Überdiagnosen lassen sich nur für Populationen durch Studien nachweisen, nicht für eine Einzelperson, da man nicht von vornherein weiss, ob noch Symptome der Erkrankung auftreten werden [35]. Autopsiestudien zeigen, dass subklinische Krankheiten bei vielen Menschen vorhanden sind, ohne dass sie je entsprechende Symptome entwickelt hätten. So wurden bei 30 bis 70 Prozent der über 60-jährigen Männer kleine, sehr langsam wachsende Prostatatumore gefunden $[6,36$, 38, 39]. Hinweise auf Überdiagnosen finden sich aber auch in Statistiken: Als das Screening eingeführt

Ärzte müssen Vorwürfe fürchten, wenn sie eine Untersuchung oder Behandlung unterlassen, Überdiagnosen werden nicht sanktioniert.

wurde, stieg die Inzidenz von Krebserkrankungen (z.B. Prostatakrebs) sprunghaft an, während die Sterberate konstant geblieben ist [6]. Der Anteil an Überdiagnosen wird mit Hilfe von randomisierten kontrollierten Studien sowie mit Längsschnittstudien berechnet ${ }^{5}$. Die Schätzungen des Anteils an Überdiagnosen schwanken teilweise sehr stark. Bei Brustkrebs bewegt sich dieser zwischen 1 und 54 Prozent, bedingt durch unterschiedliche Studiendesigns und Schätzmethoden, untersuchte Populationen, Häufigkeit des Screenings usw.
[40, 41]. In einer Studie zur Implementierung eines nationalen Mammographie-Screening-Programms in den Niederlanden von 1990 bis 2006 wird die Überdiagnoserate auf 2,8 bis 9,7 Prozent geschätzt [40] und in einer Cochrane Review auf 30 Prozent [42].

\section{Wie kommt es zu Overuse?}

Overuse kann durch verschiedene Einflussfaktoren entstehen. Abbildung 1 gibt eine Übersicht der wichtigsten Faktoren, welche nachfolgend erläutert werden.

\section{Medizinische und technologische Entwicklung} Überdiagnosen werden durch die folgenden medizinischen und technologischen Entwicklungen begünstigt:

- Ausweitung von Krankheitsdefinitionen:

Diagnosekriterien wurden erweitert und Schwellenwerte gesenkt, z.B. für Bluthochdruck, um negative Folgen wie einen Herzinfarkt zu vermeiden. Allerdings gelten dadurch immer mehr Personen als krank, und die Grenze zwischen Risikofaktor sowie Erkrankung verschwimmt. Dieses Phänomen wird deshalb auch als Pathologisierung gesundheitlicher Zustände (engl. disease mongering) bezeichnet. Je tiefer ein Schwellenwert gesetzt wird, desto geringer ist die Wahrscheinlichkeit für einen Nutzen der Behandlung, insbesondere in Abwägung zu den möglichen Nebenwirkungen. Ein Behandlungserfolg bei einer schweren Anomalie bedeutet nicht unbedingt, dass dieser sich auch bei einer leichten Form einstellt [6, 36, 37].

- Screening:

Ein Screening bei gesunden Personen dient dazu, Krankheiten wie Prostatakrebs möglichst früh zu
5 Mehr Informationen zu den verwendeten Methoden siehe $[36,40,41]$

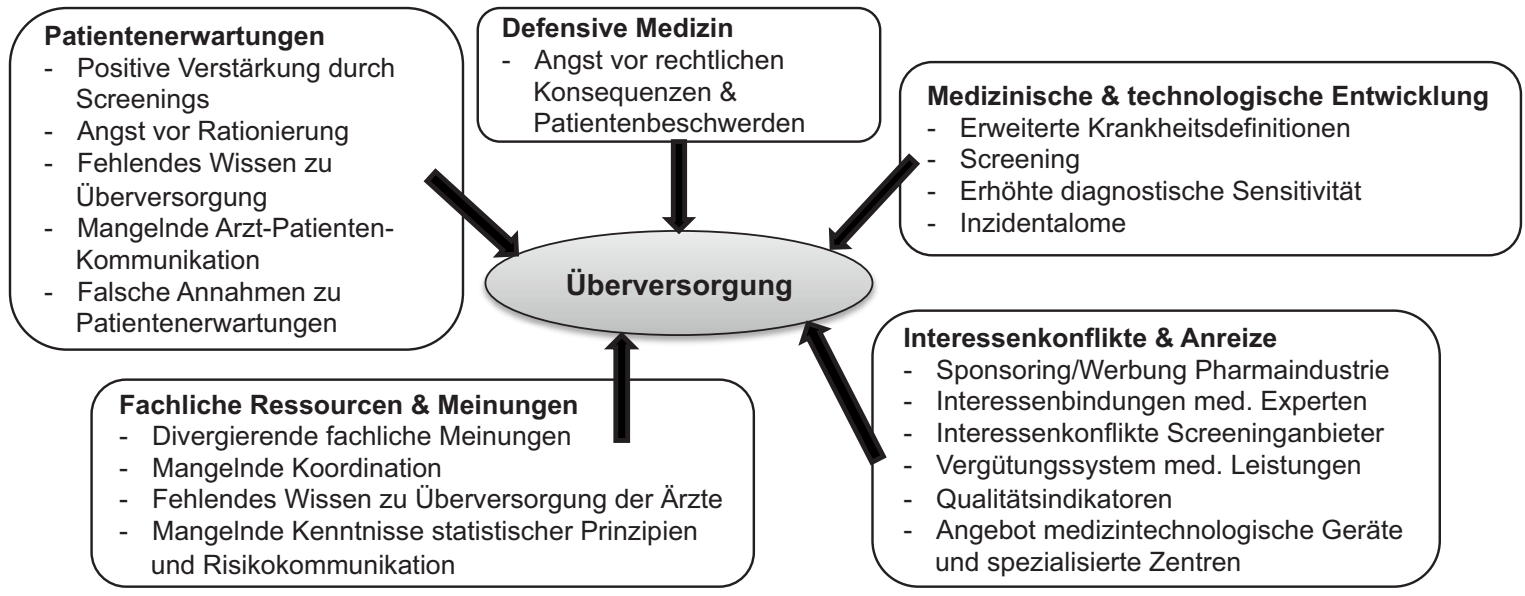

Abbildung 1: Einflussfaktoren auf Overuse. 


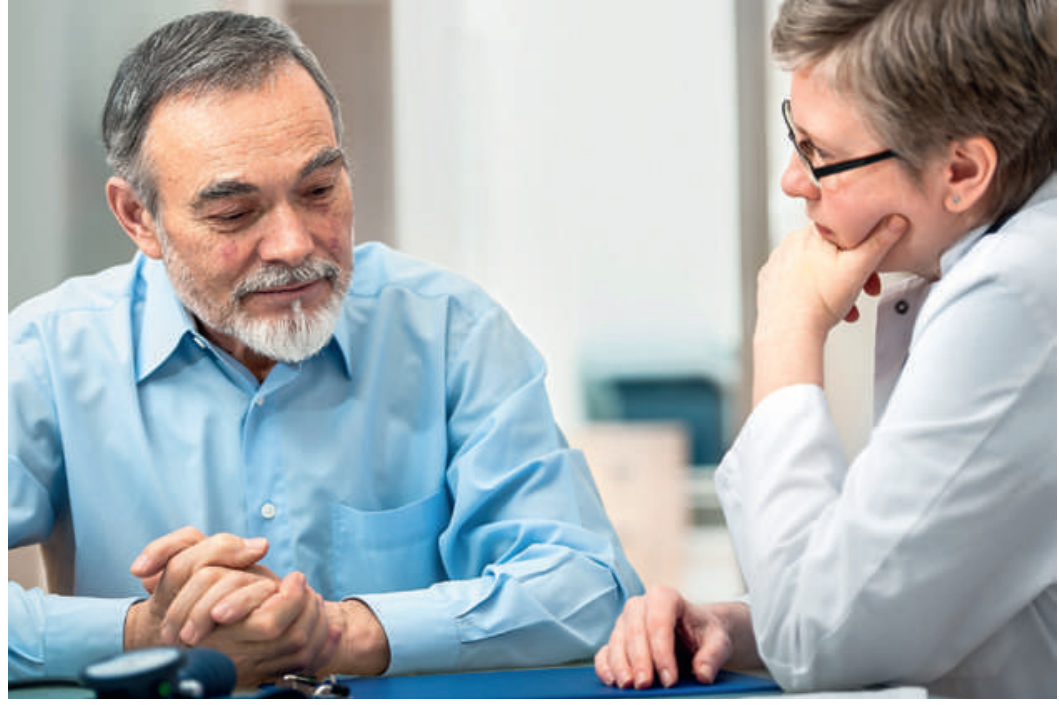

Eine US-Studie belegt, dass onkologische Patienten nur in 1 Prozent aller Konsultationen unangemessene Forderungen nach Tests oder Behandlungen stellten und Ärzte nur in 0,14 Prozent aller Konsultationen eine unnötige Intervention aufgrund von Patientenforderungen durchführten [70].

entdecken und mit einer frühzeitigen Behandlung die Mortalität zu verringern. Aber Screenings können auch zu Überdiagnosen führen, wenn sich die Krebsarten zurückbilden, unverändert bleiben oder nur ganz langsam wachsen (vgl. Abschnitt Was ist Overuse?). Die Gefahr von Überdiagnosen ist desto grösser, je intensiver nach Auffälligkeiten gesucht wird, etwa wenn Screenings sehr häufig durchgeführt werden oder in einer sehr breiten Altersgruppe [6, 36, 39].

- Zunehmende diagnostische Sensitivität und Inzidentalome:

Bildgebende Verfahren wie Computertomographien kommen immer häufiger zum Einsatz, und deren Auflösung wurde im Laufe der Zeit immer besser, so dass man heute mehr und kleinere Anomalien feststellen kann. Ausserdem werden bei diagnostischen Untersuchungen (z.B. Labortests, bildgebenden Verfahren oder genetischen Tests) manchmal zufällig Anomalien entdeckt, die nicht im Zusammenhang mit dem Untersuchungsgrund stehen, sogenannte Inzidentalome. Kleinere Auffälligkeiten führen aber seltener zu Symptomen und entpuppen sich häufiger als Überdiagnosen $[6,36,38]$.

7 Die Forschung in diesem Bereich wird unter dem Begriff «Variations in Care" geführt und beschreibt grosse Unterschiede in der Häufigkeit von medizinischen Leistungen zwischen verschiedenen Ländern [58] oder Regionen eines Landes (z.B. www.dartmouth atlas.org).

In unserem Gesundheitssystem bestehen teilweise Interessenkonflikte und Anreize, die Overuse mit sich bringen können. Die Pharmaindustrie und Medizingerätehersteller haben Interesse, ihre Produkte zu verkaufen. Je mehr Menschen als behandlungsbedürftig gelten, desto mehr profitieren sie. Produktewerbung bei Ärzten, Sponsoring von Patientenorganisationen, Kampagnen zur Früherkennung und Forschungssubventionen können Ärzte und Patienten beeinflussen [1, 6, 38, 43-45]. Ausserdem belegt eine Studie [46], dass 75 Prozent der Experten, die neue diagnostische Kriterien festlegen, Interessenbindungen zur Industrie aufweisen. Anbieter von Screenings haben ein Interesse, dass möglichst viele Menschen an einer Vorsorgeuntersuchung teilnehmen. So besteht ein Interessenkonflikt zwischen der Motivierung teilzunehmen und einer neutralen Information über Vor- und Nachteile. Keine der Informationsbroschüren von öffentlich finanzierten Mammographiescreenings in englischsprachigen und skandinavischen Ländern von 2004/05 enthielt Informationen zu Überdiagnosen [47] (vgl. Analyse in Deutschland [48]). Für die Schweiz liegt noch keine solche Analyse vor, aber vermutlich wird das Thema Overuse in neueren Informationsbroschüren eher berücksichtigt ${ }^{6}$. Mögliche Anreize für Overuse schafft aber auch das Finanzierungssystem von medizinischen Leistungen: Je mehr Untersuchungen und Behandlungen durchgeführt werden, desto höher sind die Einnahmen [4, 5052]. Bei einer Studie zu den Auswirkungen von DRGs bei Spitalärzten [53] in der Schweiz gaben 49 Prozent

\section{Überdiagnosen setzen Patienten und} ihre Familien teilweise grossen emotionalen Belastungen aus.

an, dass manchmal aus ökonomischen Gründen medizinisch unnötige Massnahmen durchgeführt wurden. Auch die Vorgaben der Versicherungen zu diagnostischen Untersuchungen für eine Kostengutsprache können einen Anreiz für unnötige Untersuchungen sein. Ebenfalls werden manchmal mit Qualitätsmassnahmen entsprechende Anreize gesetzt (ein Qualitätsindikator für eine möglichst hohe Teilnahmequote an Vorsorgeuntersuchungen [6,38] oder die Festlegung von minimalen Fallzahlen für bestimmte Operationen). Auch das Angebot, wie z.B. die Anzahl medizintechnologischer Geräte, oder spezialisierte Zentren können einen Anreiz für Overuse schaffen, da sich diese nur bei einer hohen Auslastung finanziell lohnen [50, 54]. Je höher die Ärztedichte in einem Land oder einer Region ist, umso grösser ist auch die Menge der erbrachten Leistungen und Kosten. Es ist aber unklar, inwiefern dies zu Overuse im hier definierten Sinn von unnötigen Leistungen für die Patienten führt [55-57].

\section{Fachliche Ressourcen und Meinungen}

Regionale Unterschiede bei der Behandlungshäufigkeit $^{7}$ - und vermutlich auch Overuse - stehen im Zusammenhang mit divergierenden Überzeugungen 
$\mathrm{zu}$ Indikationen [44] und einer geographisch unterschiedlichen Verbreitung von neuen Technologien oder Prozeduren. Ebenso führen divergierende Überweisungsraten an Spezialisten zu Unterschieden bei der Behandlungshäufigkeit, und eine mangelhafte Kommunikation zwischen Grundversorgern und Spezialisten respektive eine fehlende einheitliche Patientenakte kann zu unnötigen Untersuchungen führen $[32,51]$.

Ein weiterer Grund für Overuse ist das lückenhafte Wissen vieler Ärzte zum Thema Overuse. Als Ergebnis einer Studie in Deutschland kam heraus, dass nur 7 von 20 Gynäkologen die Wahrscheinlichkeiten eines Nutzens von Brustkrebsscreening kennen. Keiner nannte unnötige Behandlungen als mögliches Risiko [59]. Um

\section{Der Einfluss der Patientenerwartungen auf Overuse wird vermutlich eher überschätzt.}

die Nützlichkeit einer Intervention abschätzen zu können, ist es zudem wichtig, dass Ärzte statistische Prinzipien richtig verstehen und in der Risikokommunikation $^{8}$ geübt sind. Beispielsweise sollten Informationen zur Sterblichkeitsreduktion immer in absoluten und nicht in relativen Prozentzahlen angegeben und die Begriffe Überdiagnose-Bias sowie Lead-time-Bias bei der Interpretation von Mortalitätsstatistiken angewandt werden $[6,36,61]$.

\section{Patientenerwartungen}

Viele Patienten (und zum Teil auch Ärzte) glauben intuitiv, dass mehr und neuere medizinische Leistungen ein Qualitätsanzeichen sind. Es fällt ihnen schwer, einfach nur den weiteren Krankheitsverlauf abzuwarten [20,54]. Medikamenten stehen Patienten teilweise sehr kritisch gegenüber [62], während Screenings sehr positiv beurteilt werden, da diese als eine Möglichkeit gesehen werden, selbst aktiv etwas für die eigene Gesundheit zu tun. Die Vorstellung, ohne Symptome eine Krankheit in sich tragen zu können, löst Angst aus, die nach einem unauffälligen Screeningbefund oder nach einem falsch positiven Befund durch Erleichterung abgelöst wird. Falls tatsächlich eine Erkrankung eintritt, ist der Patient froh, aufgrund des Screenings rechtzeitig behandelt werden zu können. Hingegen würde er sich Vorwürfe machen, nicht am Screening teilgenommen zu haben. Patienten nehmen an, das Screening hätte einen günstigen Einfluss auf den Verlauf der Erkrankung gehabt [6, 36, 39, 62], was aber nicht unbedingt der Fall sein muss (siehe Überdiagnose-Bias [60]). Vorstösse, die Screenings einschränken möchten oder eine Behandlung gemäss Guidelines fordern, werden deshalb von Patienten eher kritisch gesehen. Dahinter steckt häufig eine Angst vor Rationierung, respektive sie befürchten dass eine sinnvolle medizinische Versorgung zu Kosteneinsparungszwecken eingeschränkt wird [62-64] oder dass Behandlungsempfehlungen ohne Berücksichtigung der individuellen Situation der Patienten umgesetzt werden. In Patientenbroschüren fehlen oft Informationen bezüglich Overuse (siehe Abschnitt Interessenkonflikte und Anreize), dementsprechend sind auch Patienten wenig über dieses Thema informiert. In einer Studie mit 317 US-amerikanischen Patienten, die zu einem Screening eingeladen worden sind [65], wurden nur 9,5 Prozent von ihrem Arzt über das Risiko von Überdiagnosen informiert. Viele Patienten möchten aber über Vor- und Nachteile informiert werden [63, 65, 66], und dieses Recht steht ihnen auch zu. Ärzte geben für das Ausbleiben eines Aufklärungsgesprächs mit dem Patienten über Nutzen und Risiken eines Screenings häufig Zeitmangel als Grund an [67].

Aber nicht nur das oft fehlende Wissen von Ärzten und Patienten führt zu Overuse, sondern auch die Interaktion zwischen Arzt und Patient. Gemäss einer US-Studie [67] führen rund 41 Prozent der Grundversorger, welche eigentlich mit einer neuen Empfehlung des US Preventive Services Task Force einverstanden waren, den PSA-Test weiterhin durch. Als Grund nannten sie häufig die Erwartung der Patienten. Eine italienische Studie [68] zeigt, dass die wahrgenommene Erwartung von Eltern bezüglich der Verschreibung von Medikamenten bei Pädiatern einen grossen Einfluss auf die tatsächliche Verschreibung von Antibiotika bei Kindern hat. Ärzte schätzen aber die Erwartungen der Patienten häufig falsch ein [69]. Der Einfluss der Patientenerwartungen auf Overuse wird vermutlich eher überschätzt. Eine US-Studie belegt, dass onkologische Patienten nur in 1 Prozent aller Konsultationen unangemessene Forderungen nach Tests oder Behandlungen stellten und Ärzte nur in 0,14 Prozent aller Konsultationen eine unnötige Intervention aufgrund von Patientenforderungen durchführten [70].

\section{Defensivmedizin}

Ärzte müssen eher Vorwürfe fürchten, wenn sie einen Test, eine Untersuchung oder Behandlung unterlassen, während Überdiagnosen nicht sanktioniert werden. Dieser Umstand führt zum Erbringen von zusätzlichen Leistungen aus Angst vor rechtlichen Klagen oder Kritik durch Patienten oder ihre Angehörigen. Dieses Ärzteverhalten wird als positive Defensivmedizin bezeichnet (z.B. unnötige Tests anordnen, diagnostische Prozeduren durchführen, Überweisungen an Spezialisten oder Spitäler [71-75]). Positive Defensivmedizin hat mit der Zeit zugenommen [73] und ist sehr verbreitet: 


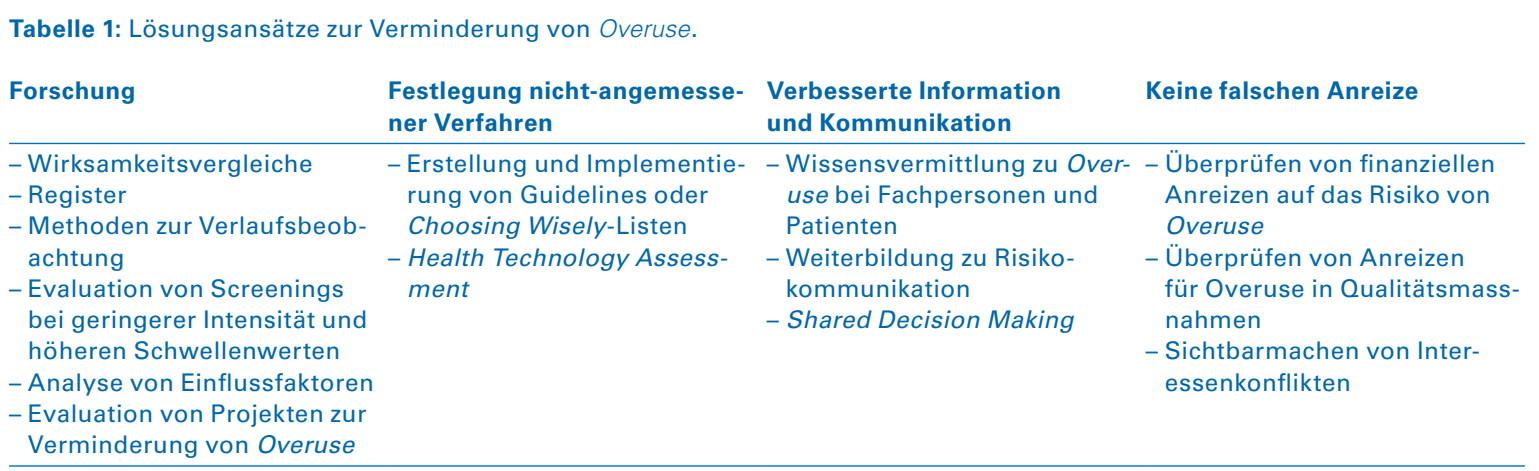

In US-Studien gaben über 90 Prozent der Ärzte in Fachgebieten mit erhöhtem Prozessrisiko an, Defensivmedizin zu praktizieren [71] ${ }^{9}$. Dabei ist unklar, ob positive Defensivmedizin tatsächlich zu weniger rechtlichen Klagen führt oder durch die zusätzlich durchgeführten Tests nicht sogar das Risiko eines Fehlers - und damit einer rechtlichen Klage - steigt [72]. In der Schweiz werden Ärzte weniger oft angeklagt als in den USA. Denn um in der Schweiz entschädigt zu werden, muss der Kläger beweisen, dass der Arzt einen Fehler gemacht hat und beim Patienten einen erheblichen $\mathrm{Ge}$ sundheitsschaden verursacht hat ${ }^{10}$. Dennoch ergab eine Befragung von Allgemeinmedizinern in der Schweiz [80], dass 43 Prozent den PSA-Test manchmal oder häufig aus rechtlichen Gründen ihren Patienten empfehlen. Dies lässt vermuten, dass Ärzte mittels Defensivmedizin nicht nur rechtliche Konsequenzen, sondern auch belastende Beschuldigungen durch Patienten vermeiden wollen [74].

9 Vgl. auch $[72,75,76]$.

10 Zudem müssen Ärztinnen und Ärzte ihrer Aufklärungspflicht, wie sie in den kantonalen Gesundheitsgesetzen verankert ist, korrekt nachkommen und dies bei Bedarf auch nachweisen können [77-79].

11 Siehe auch https://www. youtube.com/

watch?v=FqQ-JuRDkl8 für ein sehr eingängiges Video zur Choosing Wisely-Kampagne von Professor James McCormack der University of British Columbia

12 In Kanada ist die Choosing Wisely-Kampagne weit fortgeschritten, mit verschiedenen PatientenInformationsmaterialien oder mit Choosing Wisely Canada STARS, einer Kampagne von und für Medizinstudierende (www.choosingwisely canada.org).
Abschnitt Was ist Overuse?). Als besonders hilfreich gelten z.B. Studien zu Wirksamkeitsvergleichen (engl. comparative effectiveness research) [51, 84]. Nutzbringend wäre die Entwicklung von Methoden, um Veränderungen zu beobachten, ohne eine sofortige Behandlung zu beginnen $[38,39,81]$ und diese mit Hilfe von Registern zu evaluieren. Bei Screenings sollten die Auswirkungen einer geringeren Intensität (z.B. durch grössere Screeningabstände, eine eingeschränkte Altersspanne oder eine Beschränkung auf Risikopopulationen) sowie die Anhebung der Schwellenwerte für weiterführende diagnostische Untersuchungen [36, 38, 39] analysiert werden. Um Lösungsansätze voranzutreiben, braucht es mehr Wissen darüber, wie stark sich die einzelnen Einflussfaktoren auf Overuse auswirken. Wichtig sind auch Evaluationen zur Wirksamkeit verschiedener Lösungsansätze (wie z.B. die Choo-

\section{Choosing Wisely-Kampagnen}

2012 lancierte die American Board of Internal Medicine (ABIM) Foundation die Kampagne Choosing Wisely in den USA. Die Kampagne thematisierte die Frage, wie verschwendete und unnötige medizinische Leistungen vermieden werden können. Darauf haben über 70 medizinische Fachgesellschaften in den USA «Top-5-Listen» veröffentlicht. Die Listen enthielten fünf häufig durchgeführte Untersuchungen oder Behandlungen, die Ärzte und Patienten in Frage stellen sollten, da sie gemäss wissenschaftlicher Evidenz dem Patienten keinen Nutzen bringen oder sogar schaden. Ziel der Kampagne war es, Diskussionen zwischen Ärzten und Patienten in Gang zu setzen (Shared Decision Making). Deshalb wurden im Rahmen der Choosing Wisely-Kampagne für Ärzte Gesprächsführungsmodule entwickelt (siehe www.choosingwisely.org) und mit Hilfe der Konsumentenorganisation Consumer Reports Patienteninformationsmaterialien" ${ }^{11}$ zu den "Top-5-Listen» erarbeitet [90-92] Die Choosing Wisely-Kampagne hat sich international schnell verbreitet: Kanada ${ }^{12}$, Australien, Grossbritannien, Italien, Japan, Holland usw. In der Schweiz veröffentlichte die Schweizerische Gesellschaft für Allgemeine Innere Medizin (SGIM) 2014 eine erste "Top-5-Liste» unter dem Namen Smarter Medicine (siehe www.smartermedicine.ch und Selby et al. [93, 94]). 
sing Wisely-Kampagnen) in Bezug auf eine tatsächliche Reduktion von Overuse [81].

\section{Festlegung nicht-angemessener Verfahren}

Wenn die nötigen wissenschaftlichen Studien vorliegen, muss darauf basierend im Konsens für eine bestimmte Indikation festgelegt werden, welches nichtangemessene respektive angemessene Behandlungen oder Untersuchungen sind, z.B. mit der RAND Appropriateness Method, mit Guidelines («Soll-nicht-Empfehlungen») oder Choosing Wisely-Listen (vgl. Abschnitt Was ist Overuse?). Diese müssen allerdings Qualitätskriterien ${ }^{13}$ genügen, wie Transparenz bezüglich der verwendeten wissenschaftlichen Evidenz [23]. Zudem haben sie eine grössere Wirkung, wenn Kliniker an deren Erstellung oder Implementierung partizipieren konnten [85]. Eine Review [86] kommt zum Schluss, dass klinische Praxisguidelines das Potential haben,

\section{Die Ärzte sollen die Vor- und Nachteile einer Untersuchung oder Behandlung mit dem Patienten abwägen und gemeinsam eine Ent- scheidung treffen.}

unnötige Operationen zu verhindern, dass deren Impact aber stark variiert. Ob eine medizinische Leistung als Overuse gilt, wird auch im Rahmen von Health Technology Assessments (HTA) festgelegt. Mit HTA wird das Kosten-Wirksamkeits-Verhältnis verschiedener medizinischer Leistungen miteinander verglichen ${ }^{14}$. Dieses Verfahren hat nicht nur Qualitätsverbesserungen zum Ziel, sondern durch den Einbezug von ökonomischen Kriterien auch Kosteneinsparungen (Rationalisierung [64]).

\section{Information und Kommunikation}

Um Overuse zu reduzieren, reicht es aber nicht, unnötige medizinische Verfahren festzulegen. Diese Erkenntnisse müssen vermittelt und implementiert werden (mehr dazu siehe [31, 87]). Wichtig ist ausserdem eine Sensibilisierung für das Thema Overuse in der Ärzteschaft und der Bevölkerung. Medizinische Fachzeitschriften wie The Journal of the American Medical Association JAMA mit der Less is More-Artikelsammlung sowie der The BMJ Too Much Medicine-Kampagne (www.bmj.com/too-much-medicine) engagieren sich in diesem Bereich. Bekannt sind die Choosing Wiselyrespektive die Smarter Medicine-Kampagne (siehe Box). Da Patienten sich häufig im Internet zu Gesundheitsthemen erkundigen, wäre es sinnvoll, evidenzbasierte, verständliche Informationen wie Guidelines, Entscheidungshilfen, option grids, Faktenboxen [60, 62, 88] online zugänglich zu machen, damit sie sich über die
Vor- und Nachteile verschiedener Optionen inklusive des Risikos von Überdiagnosen informieren können. Die Ärzte sollen die Vor- und Nachteile einer Untersuchung oder Behandlung mit dem Patienten abwägen und gemeinsam eine Entscheidung treffen (Shared Decision Making) [85, 88]. Denn je nach Präferenz des Patienten fällt die Entscheidung unterschiedlich aus, z.B. wie viel Gewicht dem Risiko von Überdiagnosen gegeben wird [89]. Eine wichtige Voraussetzung für Shared Decision Making ist, dass Ärzte noch besser ausrespektive weitergebildet werden in Bezug auf grundlegende statistische Begriffe und die Risikokommunikation mit dem Patienten (vgl. Abschnitt Fachliche Ressourcen und Meinungen).

\section{Falsche Anreize vermeiden}

Bestehende und geplante Anreize und Strukturen sollten immer auch auf das Risiko von Overuse überprüft werden. Finanzielle Anreize (z.B. Boni) und Qualitätsmassnamen (z.B. Qualitätsindikatoren) sollten unnötige Untersuchungen oder Behandlungen nicht fördern oder das direkte Anordnen von Tests begünstigen anstelle eines Gesprächs mit dem Patienten über Vorund Nachteile [2, 6, 50, 52]. Bei der Definition von Krankheiten sollte das Risiko für Overuse mitgedacht werden. Der Eingriff in Anreize und Strukturen zur Verminderung von Overuse birgt aber immer auch das Risiko von nicht-intendierten Konsequenzen, weshalb solche Massnahmen nur sehr zurückhaltend eingesetzt werden sollten. Unbedingt ist hingegen die Offenlegung von Interessenbindungen einzufordern, sei es bei Studien, Sponsoring oder medizinischen Experten, die Richtlinien oder Krankheitsdefinitionen festlegen. Dabei ist allerdings unklar, ob diese Massnahme zu einer Reduktion von Overuse beitragen kann. Deshalb wird Ärzten geraten, PR-Informationen der pharmazeutischen Industrie zu meiden [45].

\section{Schlussfolgerungen}

Overuse ist ein Problem der Patientensicherheit, denn durch unnötige Tests, Untersuchungen und Behandlungen leidet ein Teil der Patienten an Nebenwirkungen und Komplikationen, die im Extremfall sogar zum Tod führen können. Überdiagnosen setzen Patienten und ihre Familien teilweise grossen emotionalen Belastungen aus, und Patienten können dadurch Nachteile beim Zugang zu Zusatzversicherungen erleiden. Überdiagnosen führen häufig zu weiteren Untersuchungen und unnötigen Behandlungen, die viel Zeit für Patienten und Ärzte bedeuten, welche andernorts fehlt. Trotz der Aktualität des Themas Overuse bei der Ärzteschaft und in der Politik wird nur in wenigen Berei- 
chen intensiv dazu geforscht. Der Grund ist, dass die Erhebung von Overuse anspruchsvoll ist. Eine Voraussetzung für eine Untersuchung ist die Entwicklung evidenzbasierter und anerkannter Definitionen, von nicht-angemessenen Behandlungen in den jeweiligen Fachbereichen. Dies ist nur für gewisse medizinische Leistungen möglich: Es muss eine eindeutige Evidenz betreffend fehlenden Nutzen oder Schaden für den Patienten vorliegen. Diese Definitionen können sich durch neue medizinische Techniken und Erkenntnisse ändern.

Auch Überdiagnosen sind nicht einfach zu erheben, da sie nicht für individuelle Patienten festgestellt werden können, höchstens nach deren Tod. Deshalb müssen Symptome und Mortalität mittels Langzeitstudien erhoben werden. Die dadurch gewonnenen Daten zur Häufigkeit von Überdiagnosen schwanken stark, was eine klare Information an die Patienten erschwert. Um das Problem betreffend Overuse effektiv angehen zu können, braucht es dringend mehr Forschung. Nur so können die Grundlagen für die Festlegung nicht-angemessener medizinischer Leistungen, z.B. mit Choosing Wisely-Listen, geschaffen werden. Insbesondere muss mehr Wissen zur Wichtigkeit der verschiedenen Einflussfaktoren und zur Wirksamkeit verschiedener Lösungsansätze generiert werden.

Overuse wird durch zahlreiche Einflussfaktoren begünstigt:

- Die medizinische und technologische Entwicklung, welche immer mehr Leute als krank definiert, sollte kritisch im Hinblick auf das Risiko von Überdiagnosen überprüft werden. Deshalb sollten die Vor- und Nachteile von ausgedehnten Screenings, erweiterten Krankheitsdefinitionen oder zusätzlichen, absichernden diagnostischen Untersuchungen sorgfältig abgewogen werden.

Korrespondenz: FMH/Abteilung DDQ

Elfenstrasse 18

CH-3000 Bern 15

Tel. 0313591111

Fax 0313591112
- Anreize, die die Menge an Untersuchungen ausweiten, sollten hinterfragt werden, und die Offenlegung von Interessenbindungen ist ausdrücklich einzufordern.
- Ärzteschaft und Bevölkerung sollten für das Thema Overuse sensibilisiert und darüber informiert werden. Patienten sollten Zugang zu evidenzbasierten Informationen über Vor- und Nachteile von umstrittenen Untersuchungen oder Behandlungen haben und auf die Risiken von Überdiagnosen aufmerksam gemacht werden.

- Kompetenzen in der Interpretation von statistischen Angaben, in der Risikokommunikation und in der Gesprächsführung sind in der Aus-, Weiterund Fortbildung der Ärzte zu fördern.

- Ein absicherndes Verhalten der Ärzte aus Angst vor Klagen und Beschuldigungen durch Patienten (positive Defensivmedizin) sollte durch ein Vorgehen wie Shared Decision Making abgemildert werden.

Die Herausforderung Overuse gestaltet sich je nach Fachrichtung anders und erfordert unterschiedliche Herangehensweisen [35, 37, 81]. Die einzelnen Fachgesellschaften sind deshalb gefordert, dieses Thema in ihrem Bereich anzugehen, und die kantonalen Ärzteorganisationen sowie Dachverbände, Initiativen in diesem Bereich zu koordinieren.

Eine Reduktion von Overuse kann auch helfen, Kosten einzusparen, was natürlich den Betroffenen und der ganzen Bevölkerung zugutekäme. Dennoch dürfen Kosteneinsparungen nicht das primäre Ziel sein, da dies von Patienten und Ärzten als Ausdruck von Rationierung kritisch gesehen würde. Overuse soll nicht zum Preis von verpassten Diagnosen respektive Underuse reduziert werden, sondern in erster Linie die medizinische Qualität verbessern. Empfehlungen zu unnötigen medizinischen Leistungen müssen immer bezogen auf die individuelle Situation und Präferenz des Patienten beurteilt werden und sollten gemeinsam mit dem Patienten mittels Shared Decision Making [88] entschieden werden.

Bildnachweis

() Alexraths | Dreamstime.com 


\section{Literatur}

1 Gigerenzer G. Bauchentscheidungen. Die Intelligenz des Unbewussten und die Macht der Intuition. München: Wilhelm Goldmann Verlag; 2008.

2 Malhotra A, Maughan D, Ansell J, Lehman R, Henderson A, Gray M, et al. Choosing Wisely in the UK: the Academy of Medical Roya Colleges' initiative to reduce the harms of too much medicine. Bmj. 2015;350:h2308.

3 PerryUndem Research/Communication. Unnecessary tests and procedures in the health care system. What physicians say about the problem, the causes, and the solutions. Results from a national survey of physicians. 2014. Available from: http://www.abimfoundation.org/Initiatives/Choosing-Wisely.aspx.

4 Sirovich BE, Woloshin S, Schwartz LM. Too little? Too much? Primary care physicians' views on US health care. Archives of Internal Medicine. 2011;171(17):1582-85.

5 Merçay C. Médecins de premier recours - Situation en Suisse, tendances récentes et comparaison internationale. Analyse de l'International Health Policy Survey 2015 du Commonwealth Fund sur mandat de l'Office fédéral de la santé publique (OFSP). Neuchâtel: Observatoire suisse de la santé; 2015.

6 Welch GH, Schwartz LM, Woloshin S. Die Diagnose Falle. München: Riva; 2013

7 Brodersen J, Siersma VD. Long-term psychosocial consequences of false-positive screening mammography. Ann Fam Med. 2013;11(2):106-15.

8 DeFrank J, Brewer NT. Some more evidence of long-term psychosocial harms from receiving false-positive screening mammography results. Evid Based Med. 2014;19(1):38.

9 Scheidegger D, Amstad H, Hurst S, Lehmann A, Nadig J, Sax A, et al. Nachhaltige Medizin. Positionspapier der Schweizerischen Akademie der Medizinischen Wissenschaften (SAMW). 2012.

10 Cassis I. Less is more. Wie steht es in der Schweiz mit dem Überkonsum von medizinischen Leistungen? Motion 13.3222. Curia Vista - Geschäftsdatenbank. Accessed: 21.11.2015, Available from: http://www.parlament.ch/d/suche/seiten/geschaefte.aspx?gesch id=20133222

11 Fehr J. Richtlinien für Kaiserschnitte. Motion 08.4023. Curia Vista - Geschäftsdatenbank. Accessed: 13.11.2015, Available from: http:// www.parlament.ch/d/suche/seiten/geschaefte.aspx?gesch id $=20084023$.

12 Hardegger T. Mengenausweitung bei medizinischen Eingriffen unter dem DRG-System. Postulat 13.3207. Curia Vista - Geschäftsdatenbank. Accessed: 13.11.2015, Available from: http://www.parla ment.ch/d/suche/seiten/geschaefte.aspx?gesch_id=20133207.

13 Bischof P. Kostenexplosion im Gesundheitswesen. Immer mehr unnötige Operationen? Interpellation 13.3995. Curia Vista - Geschäftsdatenbank. Accessed: 13.12.2015, Available from: http:// www.parlament.ch/d/suche/seiten/geschaefte.aspx?gesch id=20133995

14 Estermann Y. Zu häufige Herzuntersuchungen ohne vorherige Abklärung. Frage 15.5116. Curia Vista - Geschäftsdatenbank. Accessed: 13.11.2015, Available from: http://www.parlament.ch/d/suche/ seiten/geschaefte.aspx?gesch id=20155116.

15 Bundesamt für Gesundheit (BAG). Bericht Gesundheit2020. 2013. Available from: http://www.bag.admin.ch/gesundheit2020/index html?lang=de.

16 Kale MS, Bishop TF, Federman AD, Keyhani S. Trends in the overuse of amulatory health care services in the U.S. JAMA Intern Med. 2013;173(2):142-48.

17 Unterversorgung. Pschyrembel Premium Online. Walter de Gruyter $\mathrm{GmbH}$

18 Craig JC, Williams GJ, Jones M, Codarini M, Macaskill P, Hayen A, et al. The accuracy of clinical symptoms and signs for the diagnosis of serious bacterial infection in young febrile children: prospective cohort study of 15781 febrile illnesses. Bmj. 2010;340:c1594.

19 Almeida C, Rodriguez M, Skootsky S, Pregler J, Steers N, Wenger N. Cervical cancer screening overuse and underuse: patient and physician factors. Am J Manag Care. 2013;19(6):482-9.

20 Mamede S, Schmidt HG. The twin traps of overtreatment and therapeutic nihilism in clinical practice. Med Educ. 2013;48(1):34-43.

21 Thürmann PA. Weniger wäre tatsächlich mehr - die Arzneimittel versorgung alter Menschen. Z Evid Fortbild Qual Gesundhwes. 2013;107(148-152).
22 von Lutterotti N. Übertherapie am Lebensende. NZZ. 26.6.2014 Available from: http://www.nzz.ch/wissenschaft/uebertherapieam-lebensende-1.18329117.

23 Strech D. Darf es auch weniger sein? Methodische und ethische Herausforderungen bei der Verringerung von Überversorgung. Z Evid Fortbild Qual Gesundhwes. 2013;107(2):156-63.

24 Korenstein D, Falk R, Howell EA, Bishop T, Keyhani S. Overuse of health care services in the United States: an understudied problem. Arch Intern Med. 2012;172(2):171-8.

25 Überversorgung. Pschyrembel Premium Online. Walter de Gruyter $\mathrm{GmbH}$.

26 Morgan DJ, Wright SM, Dhruva S. Update on medical overuse. JAMA Intern Med. 2015;175(1):120-4.

27 Jamoulle M. Quaternary prevention, an answer of family doctors to overmedicalization. Int J Health Policy Manag. 2015;4(2):61-4.

$28 \mathrm{Kraft}$ E, Hersperger M, Herren D. Diagnose und Indikation als Schlüsseldimensionen der Qualität. Schweizerische Ärztezeitung. 2012;93(41):1485-89.

29 Institute of medicine. Crossing the quality chasm: A new health system for the 21st century - Brief report. 2001. Available from: http://www.nap.edu/books/0309072808/html/.

30 AGQ-FMH. Wirksam, zweckmässig, wirtschaftlich - Betrachtung aus ärztlicher Sicht. Schweizerische Ärztezeitung. 2007;88(40):1665-69.

31 Hostettler S, Kraft E, Bosshard C. Guidelines - Qualitätsmerkmale erkennen. Schweizerische Ärztezeitung. 2014;95(3):45-51.

32 Keyhani S, Siu AL. The underuse of overuse research. Health Serv Res. 2008;43:1923-30

33 Chmiel C, Reich O, Signorell A, Tandjung R, Rosemann T, Senn O. Appropriateness of diagnostic coronary angiography as a measure of cardiac ischemia testing in non-emergency patients - A retrospective cross-sectional analysis. PLoS One. 2015;10: e0117172. DOI: doi:10.1371/journal.pone.0117172.

34 Straumann F. Ärzte machen vorschnell teure Herzkatheter-Untersuchungen. Tages Anzeiger. 04.03.2015.

35 Hofmann B. Diagnosing overdiagnosis: conceptual challenges and suggested solutions. Eur J Epidemiol. 2014;29(9):599-604.

36 Chiolero A, Paccaud F, Aujesky D, Santschi V, Rodondi N. How to prevent overdiagnosis. Swiss Med Wkly. 2015;145:w14060.

37 Carter SM, Rogers W, Heath I, Degeling C, Doust J, Barratt A. The challenge of overdiagnosis begins with its definition. Bmj. 2015;350.

38 Moynihan R, Doust J, Henry D. Preventing overdiagnosis: how to stop harming the healthy. Bmj. 2012;344:e3502.

39 Esserman LJ, Thompson IM, Reid B, Nelson P, Ransohoff DF, Welch $\mathrm{HG}$, et al. Addressing overdiagnosis and overtreatment in cancer: prescription for change. Lancet Oncol. 2014;15(6):e234-42.

40 de Gelder R, Heijnsdijk EA, van Ravesteyn NT, Fracheboud J, Draisma G, de Koning HJ. Interpreting overdiagnosis estimates in population-based mammography screening. Epidemiol Rev. 2011;33(1):111-21.

41 Etzioni R, Gulati R, Mallinger L, Mandelblatt J. Influence of study features and methods on overdiagnosis estimates in breast and prostate cancer screening. Ann Intern Med. 2013;158(11):831-8.

42 Gotzsche PC, Jorgensen KJ. Screening for breast cancer with mammography. Cochrane Database Syst Rev. 2013;6:CD001877.

43 Kaiser T, Ewers H, Waltering A, Beckwermert D, Jennen C, Sawicki P. Sind die Aussagen medizinischer Werbeprospekte korrekt? arzneitelegramm. 2004;35.

44 Klemperer D. Über- und Unterversorgung in der Medizin. Swiss Medical Forum. 2015;15(39):866-71.

45 Spurling GK, Mansfield PR, Montgomery BD, Lexchin J, Doust J, Othman N, et al. Information from pharmaceutical companies and the quality, quantity, and cost of physicians' prescribing: a systematic review. PLoS Med. 2010;7(10):e1000352.

46 Moynihan RN, Cooke GP, Doust JA, Bero L, Hill S, Glasziou PP. Expanding disease definitions in guidelines and expert panel ties to industry: a cross-sectional study of common conditions in the United States. PLoS Med. 2013;10(8):e1001500.

47 Jorgensen KJ, Gotzsche PC. Content of invitations for publicly funded screening mammography. Bmj. 2006;332(7540):538-41

48 Kurzenhäuser S. Welche Informationen vermitteln deutsche Gesundheitsbroschüren über die Screening-Mammographie? ZaeFQ 2003;97:53-57. 
49 Swiss cancer screening. Früherkennung durch Mammografie Brustkrebs frühzeitig erkennen - Heilungschancen verbessern. 2014. Available from: http://www.swisscancerscreening.ch/filead min/user_upload/Documents/SwissCancerScreening/WWW/ Editors/Downloads/Brustkrebs/Downloads d Brustkrebs/ Brosch\%C3\%BCre_d_Fr\%C3\%BCherkennung_Mammografie.pdf.

50 McCulloch P, Nagend̄ran M, Campbell WB, Price A, Jani A, Birkmeyer JD, et al. Strategies to reduce variation in the use of surgery. The Lancet. 2013;382(9898):1130-39.

51 Birkmeyer JD, Reames BN, McCulloch P, Carr AJ, Campbell WB, Wennberg JE. Understanding regional variation in the use of surgery. Lancet. 2013;382(9898):1121-29.

52 Meyer B. Boni - die Position der FMH. Schweizerische Ärztezeitung. 2013;94(51/52)

53 Fässler M, Wild V, Clarinval C, Tschopp A, Faehnrich JA, Biller-Andorno N. Impact of the DRG-based reimbursement system on patient care and professional practise: perspectives of Swiss hospital physicians. Swiss Med Wkly. 2015;145:w14080.

54 Hofmann BM. Too much technology. Bmj. 2015;350:h705.

55 Leonard C, Stordeur S, Roberfroid D. Association between physician density and health care consumption: a systematic review of the evidence. Health Policy. 2009;91(2):121-34.

56 Trageser J, Vettori A, Iten R, Crivelli L. Effizienz, Nutzung und Finanzierung des Gesundheitswesens. Bern: Akademien der Wissenschaften Schweiz; 2012

57 Davis P, Gribben B, Scott A, Lay-Yee R. The "supply hypothesis" and medical practice variation in primary care: testing economic and clinical models of inter-practitioner variation. Social Science \& Medicine. 2000;50:407-18.

58 OECD. Geographic variations in health care: What do we know and what can be done to improve health system performance? 2014. DOI: 10.1787/9789264216594-en.

59 Wegwarth O, Gigerenzer G. «There is nothing to worry about»: gynecologists' counseling on mammography. Patient Educ Couns. 2011;84(2):251-6.

60 Gigerenzer G, Kuoni J, Rischard R. Was Ärzte wissen müssen. Swiss Medical Forum. 2015;15(36):787-93.

61 Wegwarth O, Gigerenzer G. Statistical illiteracy in doctors. In G Gigerenzer, Gray MJA, Editors. Better doctors, better patients, better decisions. Envisioning health care 2020. Cambridge: The MIT Press; 2011. p. 137-51.

62 Schleifer D, Rothman DJ. «The ultimate decision is yours»: exploring patients' attitudes about the overuse of medical interventions. PLoS One. 2012;7(12):e52552.

63. Hersch J, Jansen J, Barratt A, Irwig L, Houssami N, Howard K, et al. Women's views on overdiagnosis in breast cancer screening: a qualitative study. Bmj. 2013;346:f158

64 Strech D. Der Abbau von Überversorgung als Teil der ärztlichen Berufsethik. Konzeptionelle Klärung und neue Perspektive. Z Gerontol Geriat. 2014;47:17-22

65 Wegwarth O, Gigerenzer G. Overdiagnosis and overtreatment Evaluation of what physicians tell their patients about screening harms. Jama. 2013;173(22):2086-87.

66 Waller J, Douglas E, Whitaker KL, Wardle J. Women's responses to information about overdiagnosis in the UK breast cancer screening programme: a qualitative study. BMJ Open. 2013;3(4).

67 Pollack CE, Noronha G, Green GE, Bhavsar NA, Carter HB. Primary care providers' response to the US Preventive Services Task Force draft recommendations on screening for prostate cancer. Arch Intern Med. 2012;172(8):668-70.

68 degli Atti MLC, Massari M, Bella A, Boccia D, Filia A, Salmaso S, et al. Clinical, social and relational determinants of paediatric ambulatory drug prescriptions due to respiratory tract infections in Italy. Eur J Clin Pharmacol. 2006;62:1055-64.

69 Moro ML, Marchi M, Gagliotti C, Di Mario S, Resi D, the «Progetto Bambini a Antibiotici « Regional G. Why do paediatricians prescribe antibiotics? Results of an Italian regional project. BMC Pediatrics. 2009;9:69-69.

70 Gogineni K, Shuman KL, Chinn D, Gabler NB, Emanuel EJ. Patient demands and requests for cancer tests and treatments. JAMA Oncology. 2015;1(1):33-39.
71 Studdert DM, Mello MM, Sage WM, et al. Defensive medicine among high-risk specialist physicians in a volatile malpractice environment. Jama. 2005;293(21):2609-17.

72 O'Leary KJ, Choi J, Watson K, Williams MV. Medical students' and residents' clinical and educational experiences with defensive medicine. Acad Med. 2012;87(2):142-8.

73 Summerton $N$. Trends in negative defensive medicine within general practice. Br J Gen Pract. 2000;50(456):565-6.

74 Cunningham W, Wilson $\mathrm{H}$. Republished original viewpoint: complaints, shame and defensive medicine. Postgrad Med J. 2011;87(1034):837-40.

75 Ortashi O, Virdee J, Hassan R, Mutrynowski T, Abu-Zidan F. The practice of defensive medicine among hospital doctors in the United Kingdom. BMC Med Ethics. 2013;14:42

76 Sathiyakumar V, Jahangir AA, Mir HR, Obremskey WT, Lee YM, Apfeld JC, et al. The prevalence and costs of defensive medicine among orthopaedic trauma surgeons: a national survey study. J Orthop Trauma. 2013;27(10):592-98.

77 BGE $117 \mathrm{Ib} 197$

78 Zimmerli U. Die Verantwortung für den Patienten im öffentlichen Spital. Schweizerische Ärztezeitung. 1991;72(15):612-17.

79 Cicoria K. Beweislastverteilung und Beweiserleichterung im Arzthaftungsprozess. Jusletter. 12. April 2010

80 Steurer J, Held U, Schmidt M, Gigerenzer G, Tag B, Bachmann LM. Legal concerns trigger prostate-specific antigen testing. J Eval Clin Pract. 2009;15(2):390-2.

81 Macdonald H, Loder E. Too much medicine: the challenge of finding common ground. Bmj. 2015;350(h1163):7.

82 Schweizererische Nationalfonds. Gesundheitsversorgung. Nationales Forschungsprogramm. Ausschreibung. 2015. Available from http://www.nfp74.ch/SiteCollectionDocuments/Call_Gesundheitsversorg de.pdf.

83 Schweizerische Akademien der Medizinischen Wissenschaften. Stärkung der Versorgungsforschung in der Schweiz. Vol. 9. 2014. Available from: http://www.samw.ch/dms/de/Publikationen/ Positionspapiere/Konzept_Versorgungsforschung.pdf.

84 Rodondi N. «Less is more» oder: Könnten wir unseren Patienten Untersuchungen oder Behandlungen ersparen? Schweiz Med Forum. 2013;13(10):196

85 Vodicka TA, Thompson M, Lucas P, Heneghan C, Blair PS, Buckley DI, et al. Reducing antibiotic prescribing for children with respiratory tract infections in primary care: a systematic review. The British Journal of General Practice. 2013;63(612):e445-e54.

86 Reames BN, Shubeck SP, Birkmeyer JD. Strategies for reducing regional variation in the use of surgery: a systematic review. Ann Surg. 2014;259(4):616-27.

87 Prior M, Guerin M, Grimmer-Somers K. The effectiveness of clinical guideline implementation strategies - a synthesis of systematic review findings. Journal of Evaluation in Clinical Practice. 2008;14(5):888-97.

88 Gerber M, Kraft E, Bosshard C. Shared Decision Making - Arzt und Patient entscheiden gemeinsam. Schweizerische Ärztezeitung. 2014;95(50):1883-89.

89 Van den Bruel A, Jones C, Yang Y, Oke J, Hewitson P. People's willingness to accept overdetection in cancer screening: population survey. Bmj. 2015;350(h980):12.

90 ABIM Foundation. Choosing Wisely. Accessed: 23.10.2015, Available from: http://www.choosingwisely.org/.

91 Morden NE, Colla CH, Sequist TD, Rosenthal MB. Choosing wisely - the politics and economics of labeling low-value services. $\mathrm{N} \mathrm{Engl}$ J Med. 2014;370(7):589-92

92 Admon AJ, Cooke CR. Will Choosing Wisely ${ }^{\circledR}$ Improve Quality and Lower Costs of Care for Patients with Critical Illness? Ann Am Thorac Soc. 2014;11(5):823-27.

93 Selby K, Gaspoz JM, Rodondi N, Neuner-Jehle S, Perrier A, Zeller A, et al. Creating a list of low-value health care activities in Swiss primary care. JAMA Intern Med. 2015;175(4):640-2.

94 Selby K, Cornuz J, Neuner-Jehle S, Zeller A, Rodondi N, Meier CA, et al. «Smarter Medicine»: 5 Interventionen, die in der ambulanten allgemeinen inneren Medizin vermieden werden sollten. Schweizerische Ärztezeitung. 2014;95(20):769-70. 